\title{
Stadt, Land, Berg \\ Vom Zusammenspiel von Dialektwahrnehmung und \\ Topographie
}

Christian Schwarz (Münster) und Philipp Stoeckle (Zürich)

\begin{abstract}
In traditional dialectology, prominent geographical conditions are particularly made responsible for linguistic divergence between neighboring dialect areas (e. g. rivers, mountain ranges, etc.). Also modern national borders, territories of the Middle Ages or even older borders between tribal areas are made responsible as factors which consider linguistic areas as "mirror pictures of history" (Bach 1950: 31, translation: CS). Geographical as well as political borders seem to function as points of orientation that speakers use for constructing their cognitive surroundings and which consequently can result in linguistic divergence.

In our contribution, we want to focus on the question of how the topographic nature of landscapes influences the mental construction of linguistic areas by lay speakers. For pursuing this question, we will discuss results from German dialect areas that cover a broad range of different topographic landscape forms. The analyzed areas are located in the utter southwest of Germany (characterized by flat lands along the Rhine valley and low mountain ranges of the Black Forest) and in South Tyrol in the very north of Italy (characterized by mountains and deep valley cuttings). In our contribution, we will argue that speakers in their subjective dialect perception orient themselves toward national borders and topographically prominent points. With regard to topography primarily valleys are used as eponyms for subjective dialect areas.
\end{abstract}

\section{$1 \quad$ Einleitung}

In den letzten Jahren hat sich innerhalb der Soziolinguistik die Ethnodialektologie (auch Laien- oder Wahrnehmungsdialektologie; engl. folk linguistics) als Forschungsrichtung etabliert. Sie beschäftigt sich mit der Frage, welche Sicht Nicht-Linguisten auf Sprache und Sprachvariation haben, nach welchen Kriterien sie Varietäten klassifizieren und wie sie diese beschreiben und bewerten. Dabei wird von der Grundannahme ausgegangen, dass Sprachgebrauch, metasprachliches Wissen und Einstellungen in einem Verhältnis zueinander stehen und laienlinguistische Konzepte und Spracheinstellungen einen Einfluss auf den Sprachgebrauch und somit auch auf den sprachlichen Wandel haben können. Erste Studien zur geographischen Verortung von Dialektgebieten durch linguistische Laien lassen sich zwar schon in der Mitte des 20. Jahrhunderts ausmachen (cf. etwa Weijnen 1946 zum Niederländischen oder Sibata 1959/1999 zum Japanischen), jedoch wurde deren Relevanz im Kontext soziolinguisti- 
scher Fragestellungen erst von Dennis Preston erkannt, der schließlich die folk linguistics als eigenständiges Forschungsgebiet innerhalb der Soziolinguistik etablierte. Auf der Grundlage von Prestons (1982, 1999) Forschungsprogramm wurden zahlreiche Studien im angloamerikanischen Raum (u. a. Inoue 1996, Montgomery 2006, Evans 2011, Cukor-Avila et al. 2012) durchgeführt, die ihrerseits wiederum in den letzten Jahren einen Boom ethnodialektologischer Arbeiten im deutschsprachigen Raum auslösten. Der geographische Fokus dieser Studien liegt dabei meist auf großräumigen Dialektlandschaften, die entweder subjektive Konzeptualisierungen in Bezug auf den gesamten deutschen Sprachraum (cf. Lameli/Purschke/Kehrein 2008, Hundt/Palliwoda/Schröder 2015) oder auf einzelne Dialekträume bzw. Bundesländer (cf. Anders 2010, Purschke 2011) zum Gegenstand haben. Dabei weisen die Ergebnisse darauf hin, dass sich die Informanten insbesondere an Bundesländern (Bairisch, Hessisch, Sächsisch), Städten (Berlinerisch, Kölsch), landschaftlich-kulturellen Charakteristika (Norddeutschland) und weiteren räumlich ausgeprägten Ankerpunkten orientieren (cf. Lameli/Purschke/Kehrein 2008; Hundt/Palliwoda/Schröder 2015). Häufig handelt es sich dabei um Wissen, das nicht durch unmittelbare Erfahrung mit der betreffenden Umgebung bzw. aufgrund von Ortskenntnis erworben, sondern in irgendeiner Form medial vermittelt wurde.

Im Gegensatz zu den auf dialektale Großräume hin orientierten Studien ist die subjektive Verortung von kleinräumigen, regionalen Dialekten bisher noch wenig untersucht worden. Neben der Untersuchung zum Alemannischen von Stoeckle (2014), aus der ein Teil der hier verwendeten Daten stammt, kann als ein Vertreter einer solchen kleinräumigen Studie das Ländere ${ }^{n}$-Projekt erwähnt werden, das die ethnodialektologische Klassifikation der sogenannten Urschweiz zum Thema hat (cf. Christen et al. 2015). Auch die hier vorgelegte Untersuchung wird sich geographisch auf kleinere Räume konzentrieren und dabei der Frage nachgehen, an welchen Faktoren sich Sprecher bei der subjektiven Konstruktion von Dialektgebieten auf der regionalen/lokalen Ebene orientieren. Es geht um die Frage, welchen Einfluss topographisch sehr unterschiedliche Gebiete auf die kognitive Konstruktion von Sprachräumen haben können. Hierzu sollen aktuelle Untersuchungsergebnisse aus Dialektgebieten vorgestellt werden, die eine große Bandbreite unterschiedlicher Landschaftsformen abdecken. Bei den analysierten Gebieten handelt es sich einerseits um den Südwesten Baden-Württembergs, der durch flaches Land (Rheintal) sowie hügeliges (Wald)gebiet und Mittelgebirge (Kaiserstuhl, Schwarzwald) geprägt ist (cf. Stoeckle 2014), andererseits um die Autonome Provinz Bozen-Südtirol, die sich in erster Linie durch alpine Gebirgslandschaft mit tiefen Taleinschnitten auszeichnet.

\section{Forschungsüberblick}

Recherchiert man zum Zusammenhang von dialektaler Divergenz und topographischen Eigenschaften des Raumes, so wird offensichtlich, dass in der Dialektologie diesem Zusammenhang von jeher hohe Relevanz zugesprochen wurde. Der gängigen Ansicht zufolge lag die wichtigste Ursache für Dialektgrenzen in erschwerten oder unterbrochenen Kommunikationsverhältnissen, die wiederum auf außersprachliche Grenzen zurückgeführt wurden (cf. Paul 1880/1975: 40-41, Bohnenberger 1928, Bach 1934/1950). An topographischen Objekten ausgerichtete Isoglossenbündel, wie beispielsweise die sog. „Schwarzwald-Schranke“ (cf. Maurer 1942: 209-213), oder der Lech (cf. König 2001) 
bestätigen die Bedeutung naturräumlicher Größen für den Verlauf von Dialektgrenzen. Im Hinblick auf die Bedeutung von Flüssen hat Pickl (2013: 146-148) mithilfe statistischer Methoden jüngst überprüft, in welchem Maß diese naturräumliche Größe mit sprachlichen Divergenzen übereinstimmt. In seinem Untersuchungsgebiet (Bayerisch-Schwaben) erhält er im Ergebnis lediglich für die zwei größten der elf analysierten Flüsse signifikante Ergebnisse (Lech und Donau), womit Flüsse als naturräumliche Faktoren sprachlicher Divergenz eher eine untergeordnete Rolle zu spielen scheinen.

Neben den naturgegebenen Eigenschaften der Erdoberfläche werden in der Forschungsliteratur auch Artefakte als erklärende Faktoren herangezogen. Es handelt sich dabei besonders um nationalstaatliche Grenzen der Neuzeit, politische Territorien des Mittelalters oder noch ältere Stammesgrenzen, die Sprachlandschaften als „,Spiegelbilder der Geschichte" formen (cf. Bach 1934/1950: 31). Als einer der ersten Dialektologen konnte Karl Haag im Rahmen seiner Kartierungsarbeiten Ende des 19. Jahrhunderts eine starke Übereinstimmung von dialektalen Isoglossen und politischen Grenzen feststellen (Haag 1898: 4). Statistisch gesichert hat diesen Eindruck in jüngster Zeit Pickl (2013: 148-154), dessen Analysen darauf hinweisen, dass für das Untersuchungsgebiet Bayerisch-Schwaben politische Grenzen dann für sprachliche Divergenz signifikant werden, wenn sie mit älteren Vorläufergrenzen zusammenfallen. Dabei erreichen Grenzen, die (inklusive ihrer in etwa deckungsgleichen Vorläufergrenzen) jünger als ca. 200 Jahre sind, keine signifikanten Werte.

Dass topographische oder menschengemachte Hindernisse im Raum nicht per se zu sprachlicher Divergenz führen, hat besonders Auer (2004) deutlich gemacht. Ihm zufolge dienen Objekte im Raum für die Sprecher als Orientierungspunkte, die sie als kognitive Konstrukte aufbauen und entlang derer sich in der Folge faktische sprachliche Divergenz ergeben kann. Nur weil also ein breiter Fluss ein Dialektkontinuum durchzieht, folgt daraus nicht, dass sich entlang dieses räumlichen Objekts sprachliche Divergenz ergibt. Dies geschieht erst durch die Entwicklung subjektiver Einstellungen von Alterität dies- und jenseits des Flusses und über die Wahl des Flusses als „Demarkationslinie“ durch die hier lebenden Menschen. Dementsprechend kann sprachliche Divergenz somit als eine Folge sozialer Alteritätskonstruktionen angesehen werden. Neuere empirische Studien liefern vermehrt Hinweise auf die Entstehung bzw. Konsolidierung von Dialektgrenzen, die in engem Zusammenhang mit Ideologien und Einstellungen stehen (cf. Streck 2012, Hansen-Morath/Stoeckle 2014, Auer et al. 2015).

Wie die bisherigen Ausführungen verdeutlichen, konnte ein Zusammenhang zwischen dialektaler Divergenz und physischen - d. h. topographischen und politischen - sowie mentalen Grenzen in der Dialektologie wiederholt nachgewiesen werden. Weniger erforscht ist bislang jedoch, wie die mentalen Räume selbst beschaffen sind. Auf der Grundlage bisheriger Studien lässt sich verallgemeinernd feststellen, dass Dialektgebiete meist anhand auffälliger räumlich-struktureller Charakteristika mental konstruiert werden, wobei neben dem Erhebungsinstrument selbst - also der vorgelegten Grundkarte ${ }^{1}$ und der darauf

\footnotetext{
${ }^{1}$ Ein weiteres Verfahren zur Elizitierung mentaler Dialekträume ist die sog. Pilesort-Methode (cf. Anders 2010, Hundt/Palliwoda/Schröder 2015), bei der die Informanten mit Städtenamen bedruckte Karten nach deren dialektaler Ähnlichkeit sortieren sollen. Da bei dieser Methode die Geographie eine untergeordnete Rolle spielt, soll an dieser Stelle nicht weiter darauf eingegangen werden.
} 
abgebildeten Informationen - sowohl der geographische Ausschnitt (und somit die Größe des Untersuchungsgebiets und der erfragten Dialektraumkonzepte) als auch die Lage eine entscheidende Rolle spielen. In Bezug auf die Verortung dialektaler Großräume innerhalb der Bundesrepublik Deutschland unterscheiden etwa Lameli/Purschke/Kehrein (2008) zwischen vier Konzepttypen, die Purschke (2011: 175) als „geographisch-politisch [...] (Bairisch), ortsbezogen[...] (Berlinerisch), geographisch-kulturell[...] (Mischtyp: Norddeutsch, Rheinisch)“ und „sprachbezogen[...] (Hochdeutsch)“ zusammenfasst. Anders (2010) bezieht sich in ihrer Untersuchung zur Wahrnehmung des Obersächsischen auf Lynchs (1960) Typologie kognitiver Kartierungsschemata, ${ }^{2}$ anhand derer sie eine Klassifikation der von ihr erhobenen handgezeichneten Dialektkarten in die zwei Kartentypen „Knotenpunktwissen (Merkzeichen, Brennpunkte, Bereiche)“ (Anders 2010: 185) und „Streckenwissen (Wege, Grenzlinien)“ (Anders 2010: 192) vornimmt. Zum ersten Typ gehören beispielsweise Karten, in denen eine Orientierung der Informanten an Städten als Dialektzentren deutlich wird, während die Karten des zweiten Typs eine Ausrichtung an (größtenteils politischen) Grenzen erkennen lassen. Die Autoren beider Studien weisen zudem darauf hin, dass Art und Anzahl der eingezeichneten Mental Maps ,von der vorgegebenen Ausgangskarte determiniert zu sein [scheinen], d. h. auf welche Rauminformationen der Informant zurückgreifen kann“" (Anders 2010: 193).

Bemerkenswert ist, dass topographische Bezüge weder auf der geographischen Makro-Ebene (Lameli/Purschke/Kehrein 2008) noch auf der Meso-Ebene (Anders 2010) eine besondere Rolle für die mentale Dialektraumkonstruktion zu spielen scheinen. Hingegen zeigen die Ergebnisse von Stoeckle (2014) zur Verortung dialektaler Kleinräume einen deutlichen Zusammenhang zwischen subjektiv empfundenen Dialektgebieten und topographischen Strukturen. Auch Purschke (2011: 175) weist darauf hin, ,dass die Nutzbarmachung einzelner Strukturierungsmuster, etwa des topographischen Bezugs, von der Projektionsfläche (großregional, regional, lokal) abhängt"“.

Bei allen Unterschieden, die sich hinsichtlich der räumlich-strukturellen Referenzen zwischen den Studien verschiedener geographischer Größenordnungen ergeben, scheinen jedoch politische (und dabei vor allem nationalstaatliche) Grenzen einen festen Orientierungspunkt als mentale Dialektgrenzen darzustellen. Dies mag einerseits in der Sache selbst begründet liegen, also in der Tatsache, dass Staatsgrenzen unabhängig von der Größe des untersuchten Gebiets kategorisch als Dialektschranken wahrgenommen werden, andererseits könnte es auch ein methodisches Artefakt sein, da die meisten ethnodialektologischen Untersuchungen ihren Fokus auf die Wahrnehmung von Dialektgebieten innerhalb eines Staates bzw. einer Teilregion legen (z. B. Anders 2010, Purschke 2011, Christen et al. 2015). Ergebnisse aus Studien in Grenzregionen (z. B. Stoeckle 2014, Auer et al. 2015, Kleene 2015, Spiekermann/Hohenstein 2016) belegen jedoch die Bedeutung von Staatsgrenzen als subjektive Begrenzungsfaktoren.

\footnotetext{
${ }^{2}$ In seiner Studie zur Wahrnehmung und Orientierung von Bewohnern der Städte Boston, Jersey City und Los Angeles entwickelt Lynch auf der Basis von Befragungen und handgezeichneten Karten eine „,ünfteilige [...] Typologie visueller Elemente kognitiver Schemata in einer Stadt" (Weichhart 2008: 197), die die Elemente „Wege“ (z. B. Straßen), „Grenzlinien“ (z. B. Mauern), „Bereiche“ (z. B. Stadtviertel), „Brennpunkte“ (z. B. Kreuzungen) und „Merkzeichen“ (z. B. Schilder oder Gebäude) enthält (cf Lynch 1960: Kap. III).
} 
Der Zusammenhang zwischen subjektiv empfundenen Dialekträumen und artifiziellen oder topographischen Strukturen im Raum konnte in der Forschung also wiederholt nachgewiesen werden. Eine Differenzierung innerhalb der topographischen Strukturen fand bislang jedoch nicht statt, d. h. es ist nicht bekannt, ob verschiedene topographische Merkmale auch zu unterschiedlichen Dialektwahrnehmungen und Kategorisierungen führen. Im Folgenden soll dieser Frage anhand eines Vergleichs zwischen zwei topographisch sehr unterschiedlich gearteten Gebieten nachgegangen werden.

\section{Charakterisierung der Untersuchungsgebiete}

Die beiden Untersuchungsgebiete, die der vorliegenden Analyse zugrunde liegen, befinden sich im oberdeutschen Sprachraum. Das Oberrheingebiet ist Teil des alemannischsprachigen Raumes und umfasst nieder- und hochalemannische Dialekte. Der Raum Südtirol ist durch südbairische Dialekte charakterisiert, wie sie auch in Nord- und Osttirol sowie in Teilen Kärntens gesprochen werden. Im Süden grenzt er an das italienische Sprachgebiet.

Eine Besonderheit des Raumes Südtirol ist sein sprachpolitischer Status. So ist das Gebiet in Folge des Ersten Weltkriegs seit 1920 nicht mehr Teil Österreichs, sondern Italiens, wodurch die dortige, größtenteils deutschsprachige, Bevölkerung nunmehr eine sprachliche Minderheit darstellt. Das Deutsche gilt hier - neben dem Itaienischen - als offizielle Amtssprache und wird von etwa 70 Prozent der Bevölkerung gesprochen. Der italienischen Sprachgruppe ordnen sich ca. 25 Prozent der Bevölkerung zu, die übrigen 5 Prozent entfallen auf die Sprecher des Ladinischen - eine weitere Sprachminderheit, die ein kompaktes Gebiet im Südosten Südtirols besiedelt.

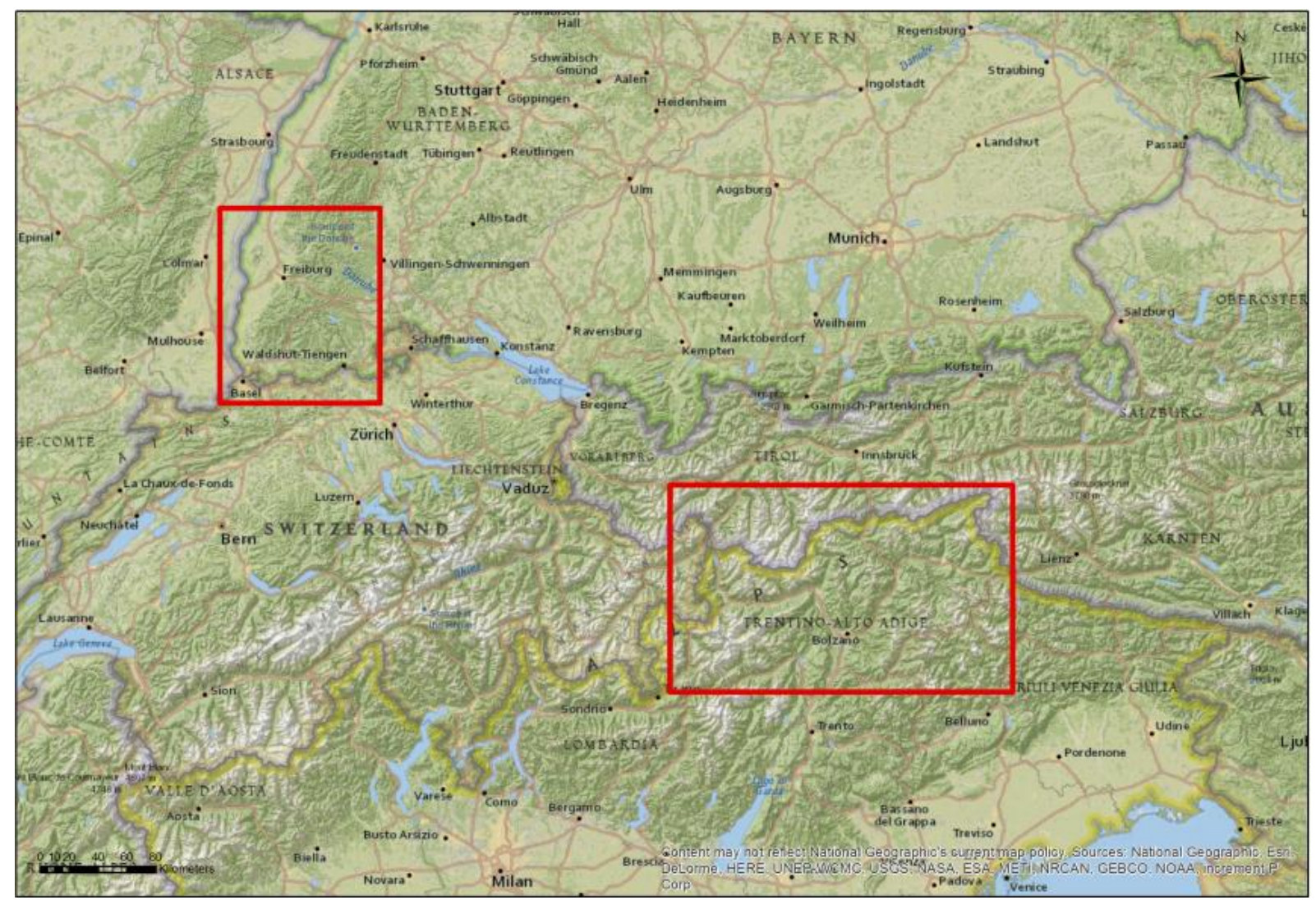

Abbildung 1: Geographische Lage der beiden Untersuchungsgebiete 
Südtirol ist die nördlichste Provinz Italiens und ist mit einer Größe von $7.400 \mathrm{~km}^{2}$ ungefähr sechs mal kleiner als die Schweiz. Das Gebiet ist südlich des Alpenhauptkammes gelegen und umfasst zu großen Teilen gebirgiges und alpines Terrain. Dies führt dazu, dass die ca. 500.000 Einwohner Südtirols das Gebiet nicht flächig bewohnen, sondern vielmehr eine Besiedlung entlang der Täler vorzufinden ist.

Der in diesem Beitrag betrachtete Teil des Oberrheingebiets liegt im äußersten Südwesten Deutschlands und grenzt im Westen an das Elsass, im Süden an die Schweiz. Sprachhistorisch bildet das Alemannische ein staatsgrenzenüberschreitendes Kontinuum, wobei neuere Studien darauf hinweisen, dass sich an den politischen Grenzen zunehmend Dialektgrenzen manifestieren (cf. Seidelmann 1989, Klausmann 1990, Schifferle 1990). Landschaftlich lässt sich das Gebiet grob in eine westliche und eine östliche Hälfte gliedern, wobei der Westen durch Flachland geprägt ist, während im Osten der Schwarzwald liegt. Insgesamt umfasst das Untersuchungsgebiet eine Fläche von ca. $4100 \mathrm{~km}^{2}$ und eine Bevölkerungszahl von etwa einer Million. Im Vergleich zu Südtirol haben wir es im Oberrheingebiet also mit einer etwa 3,5-mal höheren Bevölkerungsdichte zu tun.

Wie aus der Gegenüberstellung hervorgeht, unterscheiden sich die beiden Untersuchungsgebiete deutlich hinsichtlich Topographie, Bevölkerung und politischer Situation und eignen sich daher besonders gut für eine vergleichende Untersuchung.

\section{$4 \quad$ Methode und Daten}

Um die subjektiven Dialektverortungen der Sprecher in beiden Regionen zu elizitieren, wurde der sog. "Draw-a-map-task" (cf. Montgomery/Stoeckle 2013) verwendet. Bei dieser Methode, die ursprünglich aus der Sozialgeographie (cf. Weichhart 2008) stammt und von Preston (u. a. 1993, 2002) für die Ethnodialektologie adaptiert wurde, wird den Teilnehmern eine Karte vorgelegt, auf der sie bestimmte räumlich-geographische Konzepte - in unserem Fall Dialektgebiete - einzeichnen sollen. Diese handgezeichneten Gebiete werden in der Forschung gewöhnlich als Mental Maps oder „kognitive Karten“ bezeichnet (cf. Weichhart 2008: 170).

In Südtirol konnten für die Befragung 43 Informanten gewonnen werden. Es handelt sich dabei um fast ausschließlich weibliche (ca. 90 Prozent) Studierende der Freien Universität Bozen im Alter zwischen 19 und 63 Jahren, die aus unterschiedlichen Gebieten Südtirols stammen und zum Zeitpunkt der Erhebung dialektologisch noch nicht „,vorbelastet“ waren, d. h. über kein Lehrbuchwissen der bairischen bzw. tirolischen Dialekte verfügten. Die Erhebungen wurden während der Lehrveranstaltungen durchgeführt.

Für die Analyse der subjektiven Dialektraumkonstruktionen im Oberrheingebiet wurde eine Teilmenge des Korpus aus Stoeckle (2014) verwendet, wobei die Auswahl der Daten aus Gründen der besseren Vergleichbarkeit mit der Südtiroler Erhebung getroffen wurde. Es handelt sich um insgesamt 127 Informanten mit einem kommunikationsorientierten Berufsprofil, die aus 32 verschiedenen Orten in Deutschland stammen. Die Informanten teilen sich gleichmäßig auf zwei Altersgruppen (25-35 Jahre und 60-70 Jahre) auf. ${ }^{3}$

\footnotetext{
3 Insgesamt nahmen an der Studie 218 Personen aus 37 Orten teil. Neben den Informanten mit einem kommunikationsorientierten Berufsprofil (etwa Bankangestellte, Juristen, Versicherungskaufleute) wurden in der älteren Sprechergruppe (60-70 Jahre) an jedem Ort noch ein Mann und eine Frau aus dem landwirtschaftlich-
} 


\begin{tabular}{|l|l|l|}
\hline & Südtirol & Oberrheingebiet \\
\hline $\begin{array}{l}\text { Charakterisierung } \\
\text { Informanten }\end{array}$ & $\begin{array}{l}\text { hauptsächlich } \\
\text { Studenten }\end{array}$ & $\begin{array}{l}\text { aus 32 Orten, verschiedene Altersgruppen, ver- } \\
\text { gleichbares (kommunikationsorientiertes) Be- } \\
\text { rufsprofil }\end{array}$ \\
\hline $\begin{array}{l}\text { Anzahl Informanten } \\
\text { (bzw. Karten) }\end{array}$ & 43 & 127 \\
\hline $\begin{array}{l}\text { Anzahl eingezeichnete } \\
\text { Dialektgebiete }\end{array}$ & 406 & 848 \\
\hline
\end{tabular}

Tabelle 1: Übersicht über das Datenkorpus

Im Gegensatz zu anderen Untersuchungen, in denen die Grundkarten für den "Draw-a-maptask" häufig nur minimale Informationen (wie Länderumrisse oder Städtenamen) enthielten (cf. z. B. Preston 1993, Montgomery 2006, Anders 2010), wurden den Informanten in den beiden hier vorgestellten Studien detaillierte Karten mit Informationen zu Topographie, Städten und Gemeinden, Gewässern und Straßen vorgelegt. Diese Vorgehensweise wird dadurch begründet, dass es sich sowohl beim Oberrheingebiet als auch bei Südtirol um vergleichsweise kleine Erhebungsgebiete handelt, die bei den Informanten als zum größten Teil bekannt vorausgesetzt werden können (etwa im Gegensatz zu Erhebungen, die sich auf den gesamten deutschen Sprachraum beziehen). Da daher davon ausgegangen werden kann, dass die subjektiven Dialektraumkonzepte eher auf Alltagserfahrungen als auf medial vermitteltem Wissen beruhen, ist der Einfluss der Informationen auf der vorgelegten Karte als eher gering einzuschätzen. ${ }^{4}$ Vielmehr sollte der hohe Grad an Detailliertheit es den Teilnehmern vereinfachen, die ihnen bekannten Dialektregionen möglichst genau einzuzeichnen.

Bei dem "Draw-a-map-task" wurden den Informanten drei Aufgaben gestellt: (1) Zunächst sollten auf eine vorgelegte Karte des Untersuchungsgebiets alle bekannten Dialektgebiete eingezeichnet und nach Möglichkeit auch benannt werden. (2) Im nächsten Schritt sollten die Informanten die eingezeichneten Gebiete hinsichtlich „Sympathie“, „Ähnlichkeit zum ,eigenen’ Dialekt“" sowie „Dialektstärke“ beurteilen. Unter Dialektstärke ist dabei nicht die Vitalität des jeweiligen Dialekts zu verstehen, sondern der von den Informanten subjektiv empfundene Abstand zum Standard. (3) Zuletzt sollten von den Informanten typische linguistische Merkmale der eingezeichneten Dialekte genannt werden. In unserem Beitrag konzentrieren wir uns auf die Untersuchung der ersten Aufgabe, d. h. die subjektive Dialektverortung

\footnotetext{
handwerklichen Berufsmilieu interviewt. Für einen stichprobenartigen Vergleich jenseits der Staatsgrenze wurden zudem drei Orte aus dem Elsass sowie zwei Orte aus der Schweiz in die Studie aufgenommen. Da es sich bei den Informanten aus Südtirol durchweg um Studierende (verschiedener Altersgruppen) handelt, wurde die Auswahl für einen besseren Vergleich auf die kommunikationsorientierten Informanten beschränkt. Die Einschränkung auf die Erhebungsorte in Deutschland wurde zudem vorgenommen, da u. a. der Einfluss der Staatsgrenze untersucht werden sollte und eine zusammenfassende Analyse der Daten von Sprechern aus verschiedenen Ländern das Ergebnis verzerrt hätte.

${ }^{4}$ Eine systematische empirische Überprüfung dieser Annahme steht allerdings bislang noch aus. Die einzigen uns bekannten Untersuchungen des Einflusses verschiedener Kartentypen auf die resultierenden Mental Maps stammen von Lameli/Purschke/Kehrein (2008) und Kleene (2015). Allerdings beziehen sich diese auf die Verortung großräumiger Dialektgebiete im gesamten Gebiet der Bundesrepublik Deutschland bzw. im oberdeutschen Sprachraum.
} 
und -benennung sowie auf deren Zusammenhänge mit der Topographie der Untersuchungsgebiete.

Die Auswertung der erhobenen Daten erfolgte größtenteils mithilfe von ArcGIS (cf. ESRI s. n.), einer Anwendungssoftware aus dem Bereich der sog. Geographischen Informationssysteme (GIS). ${ }^{5}$ Dabei wurden zunächst sämtliche Karten der Informanten digitalisiert und im Anschluss die eingezeichneten Dialektgebiete in ein GIS-Vektordatenformat übertragen. Auf dieser Grundlage konnten schließlich numerische Analysen (wie die Aggregation von Dialektgebieten) durchgeführt werden, deren Ergebnisse sich in Karten (cf. Abbildungen 2 und 3) darstellen lassen. Das Ziel dieser Vorgehensweise bestand darin, über eine Gesamtdarstellung aller Daten diejenigen Gebiete zu ermitteln, die von besonders vielen Informanten als Dialektgebiete identifiziert wurden und die somit als besonders salient angesehen werden können.

Ein weiterer Auswertungsschritt bestand - neben der Aggregation der Dialektgebiete - in der quantitativen Auswertung der Dialektbenennungen (cf. Tabellen 2 und 3). Dieser Schritt erwies sich als wichtige Ergänzung zur geographischen Analyse der Daten, da die Informanten zwar mit großer Übereinstimmung die gleichen oder zumindest sehr ähnliche Benennungen für die eingezeichneten Dialektgebiete verwendeten, sich an manchen Stellen jedoch kleinere Abweichungen ergaben. Zudem kam es vor, dass ein Informant ein Gebiet einzeichnete, dieses jedoch nicht benannte (und umgekehrt).

\section{$5 \quad$ Untersuchungsergebnisse}

\subsection{Oberrheingebiet}

Aus Südwestdeutschland wurden für die Auswertung die Daten von 127 Informanten berücksichtigt. Diese zeichneten insgesamt 848 Dialektgebiete ein, d. h. durchschnittlich etwa 6,7 Gebiete pro Informant. ${ }^{6}$ Da bei der Erhebung explizit nach der Verortung des jeweils ,eigenen“ Dialekts (im Unterschied zur Verortung der bekannten ,weiteren“ Dialektgebiete) gefragt wurde und sich herausstellte, dass sich die zugrunde liegenden Strategien zum Teil systematisch voneinander unterscheiden (cf. Stoeckle 2014: 365), wurden bei der Aggregation nur die Ergebnisse für die Frage nach den ,,weiteren“ Gebieten aufgenommen. Ein weiterer Grund für diese Einschränkung besteht darin, dass die Informanten - im Gegensatz zur Erhebung in Südtirol - gleichmäßig verteilt aus 32 Orten im gesamten Untersuchungsgebiet stammen und somit die Aufnahme der jeweils ,eigenen“ Dialektgebiete zu lokalen Verzerrungen des Gesamtergebnisses geführt hätte. Das Ergebnis der Aggregation der übrigen 708 handgezeichneten Dialektgebiete ist in Abbildung 2 dargestellt.

\footnotetext{
5 Zur Verwendung Geographischer Informationssysteme in der Ethnodialektologie cf. Montgomery/Stoeckle (2013).

${ }^{6}$ Die Standardabweichung beträgt $S D=2,76$.
} 


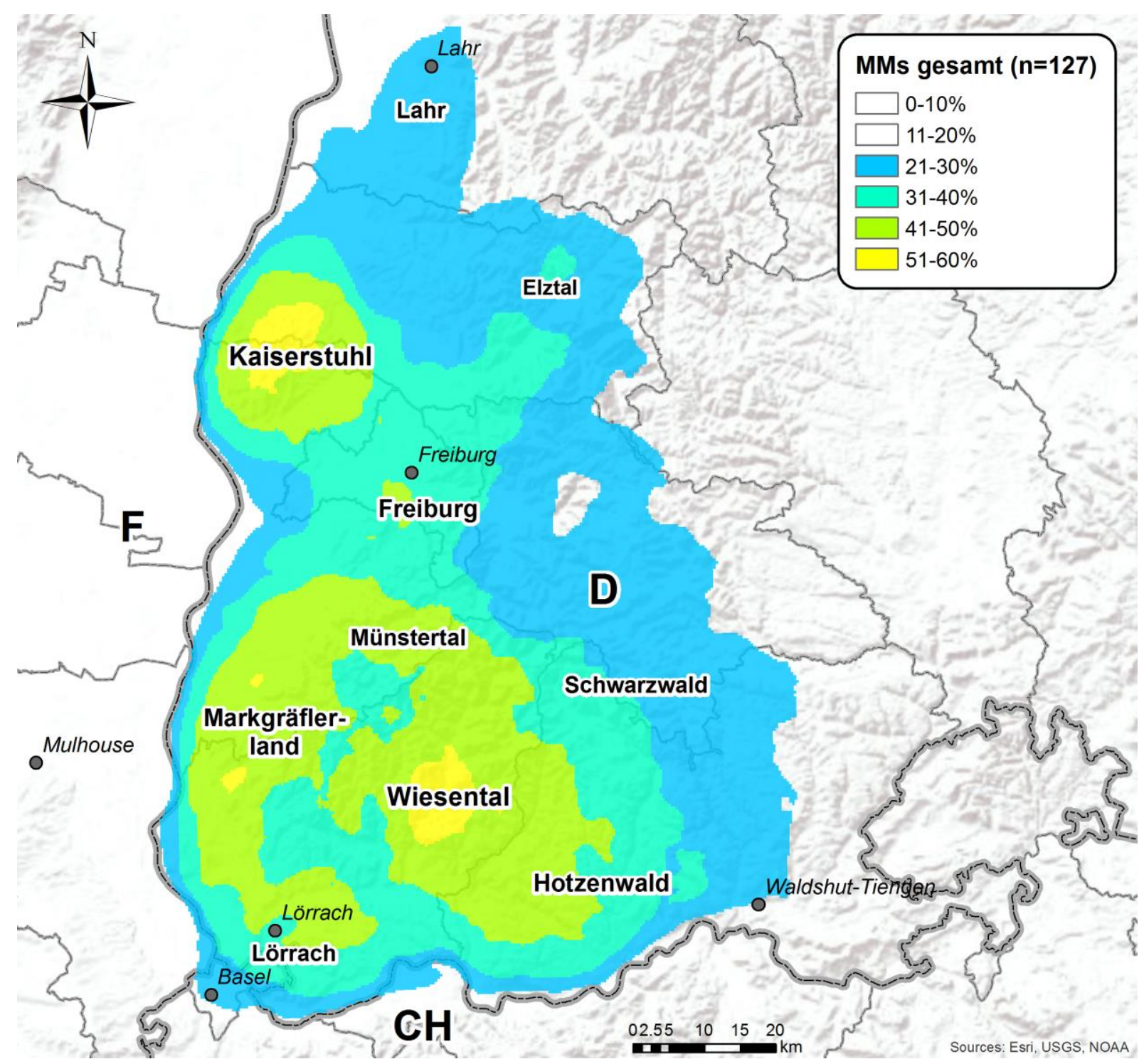

Abbildung 2: Aggregierte Darstellung der Dialektverortungen im Oberrheingebiet. Datengrundlage: 708 von den Informanten eingezeichnete Einzelgebiete.

Die verschiedenen Grade an Übereinstimmung werden durch unterschiedliche Farben dargestellt, wobei eine Einteilung in Intervalle von 10\% vorgenommen wurde. Eine blau eingefärbte Fläche bezeichnet demnach ein Gebiet, das von 21\%-30\% der Informanten (27-38) als Dialektgebiet eingezeichnet wurde. ${ }^{7}$ Zur besseren Identifikation der salientesten Dialektregionen wurden zusätzlich die Benennungen der Informanten übernommen, wie sie in Tabelle 2 wiedergegeben sind. In der Abbildung wird deutlich, dass die Informanten den Untersuchungsraum nicht gleichmäßig in Gebiete einteilen, sondern es stechen mehrere Regionen mit höherer Überlagerung eingezeichneter Dialektgebiete hervor. Besonders häufig wurden die Gebiete um das Wiesental (58\%) und den Kaiserstuhl (53\%) eingezeichnet, aber auch das Markgräflerland (51\%) und der Hotzenwald (45\%) wurden von vielen Informanten als Dialektgebiete gekennzeichnet. Weiterhin sind Häufungen von Mental Maps im Münstertal

\footnotetext{
${ }^{7}$ Weitere Charakterisierungen der Gebiete gehen nicht in die Analyse ein, d. h. es wurden alle handgezeichneten Dialektgebiete unabhängig von deren Benennung oder weiteren Attribuierungen bezüglich Sympathie etc. aufgenommen.
} 
(45\%) und im Elztal (31\%) sowie in den Umgebungen der Städte Lörrach (43\%), Freiburg (42\%) und Lahr (25\%) erkennbar.

Neben den lokalen Akkumulationen der Mental Maps lassen sich Regionen erkennen (z. B. westlich von Freiburg oder westlich des Elztals), die vergleichsweise selten als eigenständige Dialektgebiete eingezeichnet wurden. Eine mögliche Erklärung dieses Befundes liegt darin, dass diese Gebiete nicht in gleichem Maße durch räumlich-strukturelle Charakteristika hervorstechen, sondern sich vielmehr geographisch zwischen salienten Dialektgebieten befinden und somit eher als Übergangsgebiete wahrgenommen werden (cf. dazu auch Stoeckle 2014: 519). Des Weiteren geht aus der Kartierung hervor, dass Regionen, die seltener als Dialektgebiete wahrgenommen wurden, teilweise auch Randgebiete darstellen (in Bezug auf die vorgelegte Karte) und sich daher offensichtlich weniger im Fokus der Informanten befinden. Wie aus den Ergebnissen zur Region Südtirol noch ersichtlich wird (cf. Abschnitt 5.2), ist eine „Vernachlässigung“ der peripheren Gebiete jedoch kein generell feststellbares Phänomen. Die Abstufung der Dialektwahrnehmung zur Peripherie hin kann also verschiedenartig sein, endet aber abrupt an der Staatsgrenze (auch in Südtirol), die demnach als prägnante mentale Dialektgrenze angesehen werden kann. In Tabelle 2 sind die häufigsten Dialektnennungen sowie ihre Häufigkeit (d. h. ihre „Wahrnehmungsstärke“) dargestellt.

\begin{tabular}{|l|l|l|l|}
\hline Gebiet & $\begin{array}{l}\text { Anzahl } \\
\text { Nennungen }\end{array}$ & Gebiet & $\begin{array}{l}\text { Anzahl } \\
\text { Nennungen }\end{array}$ \\
\hline Kaiserstuhl & $61(48 \%)$ & Münstertal & $30(24 \%)$ \\
\hline Wiesental & $53(42 \%)$ & Lörrach & $27(21 \%)$ \\
\hline Hotzenwald & $46(36 \%)$ & Lahr/Ortenau & $25(20 \%)$ \\
\hline $\begin{array}{l}\text { Markgräflerland/ } \\
\text { Rebland }\end{array}$ & $44(35 \%)$ & (Hoch-)Schwarzwald & $25(20 \%)$ \\
\hline Freiburg & $43(34 \%)$ & Elztal & $24(19 \%)$ \\
\hline
\end{tabular}

Tabelle 2: Häufigste Dialektbenennungen im Oberrheingebiet

Vergleicht man die Häufigkeiten der Benennungen mit den Überlagerungen der eingezeichneten Gebiete, so ergibt sich ein ähnliches Bild, wobei sich die Rangfolge zum Teil unterscheidet. Generell ist festzustellen, dass die Übereinstimmungen bei den eingezeichneten Gebieten deutlich höher sind als bei den Benennungen. Dies mag einerseits darauf zurückzuführen sein, dass in der Erhebung die Zeichenaufgabe im Vordergrund stand und deshalb in der Regel nur sehr selten Dialektgebiete genannt wurden, die die Informanten nicht bereits zuvor eingezeichnet hatten. Ein anderer Grund für die Divergenz liegt sicherlich darin, dass sich beim Zeichnen viel eher Überschneidungen verschiedener Dialektraumkonzepte ergeben (d. h. dass etwa ein Informant ein Gebiet „Schwarzwald“ zeichnet, das aber gleichzeitig Teile des Münstertals oder des Wiesentals umfasst), während bei den Benennungen naturgemäß eine klarere Trennschärfe gegeben ist.

\section{$5.2 \quad$ Südtirol}

Aus der Region Südtirol stehen für die Untersuchung Karten von 43 Informanten mit insgesamt 406 eingezeichneten Dialektgebieten zur Verfügung, d. h. im Durchschnitt wurden 
pro Informant 9,4 Gebiete eingezeichnet. ${ }^{8}$ Dies sind deutlich mehr als im Oberrheingebiet, wo jeder Informant im Schnitt lediglich 6,7 Gebiete zeichnete. Wie ein Mann-Whitney- $U$-Test zeigt, ist der Unterschied statistisch höchstsignifikant. ${ }^{9}$ Bereits aus diesem Unterschied ergibt sich also ein erstes Ergebnis, das sich als höhere Dialektbewusstheit der Südtiroler Informanten im Vergleich zu denjenigen aus dem Oberrheingebiet interpretieren lässt. Ein Grund dafür ist vermutlich die prägnantere topographische Strukturierung Südtirols, die den Informanten eine deutlichere mentale Fragmentierung der subjektiven Dialekträume bietet.

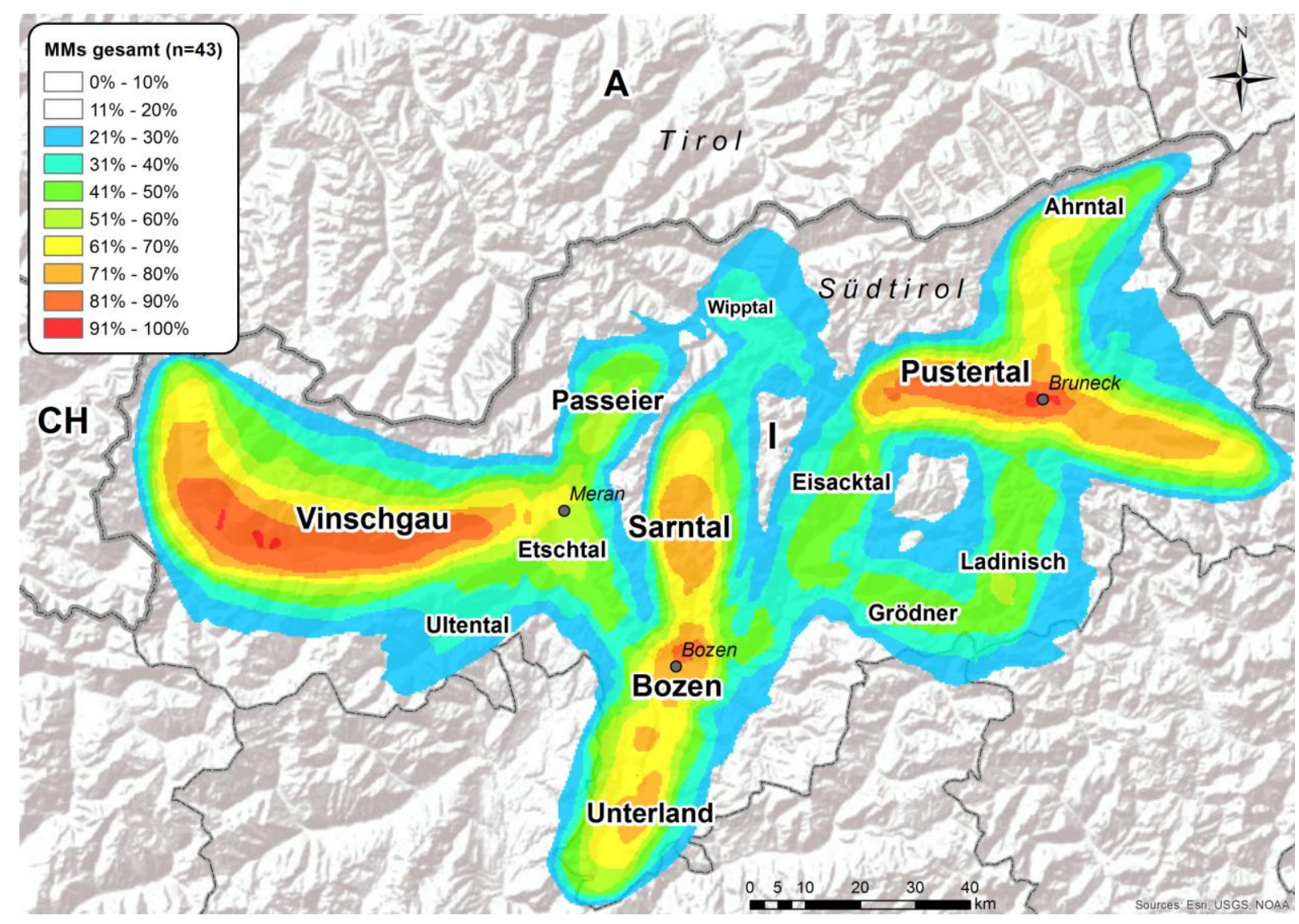

Abbildung 3: Aggregierte Darstellung der Dialektverortungen in Südtirol. Datengrundlage: 406 von den Informanten eingezeichnete Einzelgebiete.

Abbildung 3 zeigt das aggregierte Ergebnis der Dialektverortungen in der Region Südtirol. Wie zuvor wurden zur besseren Identifikation der salienten Dialektgebiete die häufigsten Benennungen (cf. Tabelle 3) hinzugefügt. Als markanteste Gebiete treten die beiden größten Täler Südtirols hervor, nämlich der Vinschgau im Westen sowie das Pustertal im Osten. Diese beiden Gebiete wurden von den Informanten mit einer Überlagerung von jeweils bis zu 91\% überaus konsistent eingezeichnet. Auch die Hauptstadt Bozen (81\%), das nördlich anschließende Sarntal (77\%) sowie das südlich angrenzende Unterland (74\%) werden von der großen Mehrheit der Informanten eingezeichnet. Eine geringere Überlagerung tritt bei Gebieten auf, die im Hinblick auf ihre Ausdehnung kleinräumiger sind oder als Übergangsgebiete zwischen den genannten Hauptgebieten liegen - so z. B. das Ahrntal

\footnotetext{
8 Die Standardabweichung beträgt $S D=3,99$.

9 Da die Voraussetzungen für einen $t$-Test (Normalverteilung, Varianzhomogenität) nicht gegeben sind, wurde ein Mann-Whitney- $U$-Test gerechnet, der folgendes Resultat erbrachte: $U=1494,5 ; Z=-4,464 ; p<0,001$.
} 
(67\%), das Etschtal (60\%) oder das Ultental (40\%). Eine Abstufung der Überlagerungen hin zur Peripherie ist, anders als im Oberrheingebiet, im Raum Südtirol nicht erkennbar. Was jedoch auch in Südtirol klar hervortritt, ist die kategorische Orientierung an den politischen Außengrenzen, an denen die Dialektverortungen und -benennungen abrupt enden. Besonders an der nördlichen Grenze ist dieser Befund erstaunlich, da die nationale Grenze hier gerade einmal ein Jahrhundert alt ist und auch im nördlich angrenzenden Tirol ebenfalls südbairische Dialekte gesprochen werden. Die Abgrenzung nach Süden geschieht offensichtlich entlang der deutsch-italienischen Sprachgrenze und ist aus linguistischer Sicht durchaus plausibel. Erstaunlich sind zudem die Ergebnisse zum Ladinischen, dessen Verbreitungsgebiet mit einer Überlagerung von bis zu 51\% eingezeichnet wird und das immerhin 30\% der Informanten benennen, obwohl es sich um keinen deutschen Dialekt, sondern um eine eigenständige romanische Sprache handelt. Dieser Befund spricht dafür, dass deutsche Dialekte bei der Verortung und Benennung offensichtlich nicht im Vordergrund stehen, sondern die Außengrenzen der Autonomen Provinz Südtirol. Dialektverortungen operieren somit nicht primär auf einer linguistischen Ebene, vielmehr scheinen zuvor politisch-nationale Kategorien wirksam zu sein.

\begin{tabular}{|l|l|l|l|}
\hline Gebiet & Anzahl Nennungen & Gebiet & Anzahl Nennungen \\
\hline Vinschgau & $40(93 \%)$ & Ahrntal & $14(33 \%)$ \\
\hline Pustertal & $39(91 \%)$ & Grödner & $14(33 \%)$ \\
\hline Bozen & $39(91 \%)$ & Ladinisch & $13(30 \%)$ \\
\hline Sarntal & $33(77 \%)$ & Ultental & $12(28 \%)$ \\
\hline Unterland & $28(65 \%)$ & Etschtal & $11(26 \%)$ \\
\hline Passeier & $21(49 \%)$ & Wipptal & $6(14 \%)$ \\
\hline Eisacktal & $16(37 \%)$ & \multicolumn{3}{|l}{} \\
\hline
\end{tabular}

Tabelle 3: Häufigste Dialektbenennungen in Südtirol

Vergleicht man hier die Häufigkeiten der Dialektbenennungen mit den Überlagerungen der eingezeichneten Gebiete, so zeigt sich ein ähnlicher Effekt wie im Oberrheingebiet, d. h. eine vergleichbare (wenn auch teilweise im Detail unterschiedliche) Rangfolge der Gebiete sowie größtenteils deutlich höhere Werte bei der Gebietsüberlagerung als bei der Benennung. Vergleicht man insgesamt die Werte der Südtiroler Informanten mit denen der Sprecher aus dem Oberrheingebiet, so wird zudem deutlich, dass die Übereinstimmungen im ersten Fall deutlich höher sind als im zweiten. Dieses Ergebnis wollen wir - neben weiteren Resultaten im folgenden Abschnitt genauer beleuchten.

\subsection{Zusammenfassung und Diskussion}

Zusammenfassend lassen sich auf der Grundlage der Ergebnisse aus dem Oberrheingebiet und Südtirol folgende räumlich-strukturelle Kategorien herausarbeiten, die für die mentale Strukturierung dieser geographischen Räume relevant sind:

(1) Die politische (besonders nationale) Zugehörigkeit stellt die deutlichste Kategorie der mentalen Verortung dar, wie im Oberrheingebiet besonders an der klaren Abgrenzung zur Schweiz und zum Elsass erkennbar ist. Auch für Südtirol darf die politisch-nationale Zugehörigkeit als stärkste Kategorie gelten, denn es werden ausnahmslos dialektale Räume 
innerhalb der politischen Grenzen der Autonomen Provinz Bozen-Südtirol eingezeichnet und die Begrenzungen nach außen genau entlang der politischen Grenzen vorgenommen. Es werden hier selbst Regionen ausgegliedert, zu denen eine äußerst enge sprachstrukturelle Verwandtschaft besteht, wie insbesondere Nord- und Osttirol. Aus Sicht der Dokumentation tirolischer Dialekte, so wie sie im Tirolischen Sprachatlas (Klein/Schmitt/Kühebacher 19651971) vorliegt, ist diese Ausgliederung nicht nachvollziehbar. Für kaum eines der untersuchten sprachlichen Phänomene stimmt die politische Grenze zu Nordtirol auch nur annähernd mit den dialektalen Grenzen überein. Für die hohe Relevanz der politischen Zugehörigkeit spricht auch die Eingliederung des bereits oben erwähnten Ladinischen in den mentalen Fokus dialektalen Umfeldes, denn aus linguistischer Sicht müsste das Ladinische als eigenständige romanische Sprache ausgegliedert oder schlichtweg ignoriert werden. Dass die Staatsgrenze nicht nur als mentale Dialektgrenze fungiert, sondern gleichzeitig den Wahrnehmungshorizont der Sprecher in Bezug auf Dialektvielfalt beschränkt, zeigt sich in beiden betrachteten Regionen durch die Tatsache, dass die eingezeichneten Regionen nicht nur an den jeweiligen Grenzen enden, sondern dass jenseits der Grenzen praktisch keine weiteren Dialektgebiete eingezeichnet werden.

(2) Städte stellen weitere mentale Orientierungspunkte dar. Hier sind für das Oberrheingebiet besonders Freiburg und Lörrach zu nennen, wobei es sich bei diesen beiden gleichzeitig um die Hauptstädte der gleichnamigen Landkreise handelt. Für Südtirol wird fast ausschließlich die Hauptstadt Bozen relevant gesetzt. Weitere Städte, wie beispielsweise Meran, Bruneck oder Brixen werden kaum oder gar nicht genannt. Offensichtlich spielen bei der Wahrnehmung von Städten also auch politische Faktoren eine wesentliche Rolle, da sie zentrale Regierungs- und Verwaltungseinheiten der sie umgebenden politischen Konstrukte (Landkreise, Provinz) sind. Dies geht auch aus den genannten linguistischen Merkmalen der Sprecher hervor. So wird sowohl Bozen als auch Freiburg und Lörrach besonders große Standardnähe attestiert (,stark an die Hochsprache angepasst“, „Vermischung mit Standardsprache“).

(3) Weiterhin treten Naturräume als topographisch determinierte Objekte auf. Im Oberrheingebiet wie in Südtirol handelt es sich dabei primär um Täler. So werden in Südwestdeutschland u. a. das Wiesental und das Münstertal genannt, in Südtirol z. B. das Pustertal, das Sarntal oder das Passeiertal. Es ließen sich an dieser Stelle zahlreiche weitere Beispiele anführen, die die Dominanz dieser Kategorie - ganz besonders in Südtirol - untermauern (cf. Abbildungen 2 und 3). Diese Kategorie darf also als die wichtigste unter den naturräumlichen Kategorien gelten, sie überragt hinsichtlich ihrer Relevanz allerdings nicht die Kategorie der politischen (nationalen) Grenzen, denn auch die Dialektgebiete außerhalb Südtirols sind durch tiefe Taleinschnitte geprägt, werden bei der Verortung von den Informanten jedoch nicht berücksichtigt. Außerdem ist in Bezug auf das Dreiländereck festzuhalten, dass hier der Kaiserstuhl als vulkanisches Kleingebirge von den Informanten als einer der salientesten Dialekträume wahrgenommen wird.

(4) Schließlich sind für das Oberrheingebiet und für Südtirol kulturell-historisch geprägte Kategorisierungen zu nennen, die allerdings als weniger präferierte Formen auftreten (besonders in Südtirol). Als Vertreter solcher Kategorisierungen können beispielweise das Markgräfler- bzw. Rebland im Oberrheingebiet oder das Burggrafenamt oder das Unterland in Südtirol angeführt werden. 
Kehren wir nun zur Ausgangsfrage zurück, wie die unterschiedliche topographische Beschaffenheit von Landschaften und die subjektive Wahrnehmung dialektaler Räume zusammenhängen. Die beiden analysierten Regionen können im Vergleich aufgrund ihrer prägnanten topographischen Verschiedenheit Aufschluss darüber geben. Welche Gemeinsamkeiten und Unterschiede zwischen den beiden Regionen lassen sich also herausarbeiten und plausibel auf den Faktor der Topographie zurückführen?

Die Gemeinsamkeiten zwischen den beiden Regionen sind vornehmlich qualitativer Art, d. h. die Informanten bedienen sich ähnlicher räumlich-struktureller Kategorien, wobei die politische bzw. die nationale Zugehörigkeit in beiden Regionen die zentrale Rolle spielt. Sowohl im Dreiländereck als auch in Südtirol endet die subjektive Verortung von dialektalen Räumen genau an den nationalen Grenzen, in Südtirol auch an den Provinzgrenzen hin zum übrigen Italien. Zwar gibt es bislang nur wenige Studien, die sich explizit mit dem Zusammenhang von Staatsgrenzen und subjektiven Dialektwahrnehmungen beschäftigen (cf. z. B. Kremer 1984; Stoeckle 2014; Auer et al. 2015), jedoch bestätigen diese sowie die Ergebnisse des vorliegenden Beitrags deren Einfluss als kategorische Begrenzungen wahrgenommener sprachlicher Ähnlichkeit bzw. Zugehörigkeit.

Die Unterschiede treten innerhalb der Untersuchungsgebiete zutage und sind im Wesentlichen quantitativer Art. So sind zwar in beiden Regionen dieselben räumlich-strukturellen Kategorien zu erkennen, doch sind diese aufgrund der Häufigkeit ihres jeweiligen Auftretens von unterschiedlicher Relevanz. Der prägnanteste Unterschied besteht hier tatsächlich hinsichtlich der Topographie. In Südtirol erfolgt durch die Sprecher bei der Dialektverortung größtenteils eine Bezugnahme auf Täler: Von den dreizehn aufgelisteten Dialektgebieten (cf. Tabelle 3) handelt es sich in elf ${ }^{10}$ Fällen um Täler, Ausnahmen bilden lediglich Bozen und das Ladinische. Im Oberrheingebiet hingegen ist eine stärkere Diversifikation zwischen den räumlichstrukturellen Kategorien zu beobachten. Unter den zehn salientesten Dialektgebieten finden sich lediglich drei Täler (Wiesental, Münstertal und Elztal), während sich die restlichen Gebiete anderen räumlichen Kategorien zuordnen lassen. Interessant ist jedoch, dass selbst bei extremer Topographie - wie in Südtirol - diese Kategorie nicht relevanter erscheint als die politisch-nationale Kategorie. Somit erscheinen nationale Artefakte bei der subjektiven Verortung sprachlicher Räume letztlich relevanter als Naturobjekte.

Ein letzter Unterschied, der zwischen den beiden Regionen deutlich hervortritt, ist die Übereinstimmung bei der Verortung bzw. Nennung von Dialektgebieten. Im Dreiländereck beträgt der Maximalwert gleicher Verortungen 58 Prozent (Wiesental), während er in Südtirol bei 91 Prozent zu liegen kommt (Pustertal und Vinschgau). Die Südtiroler sind also weit konsistenter bei der Verortung ihrer subjektiven Dialektgebiete. Diese Konsistenz könnte einerseits durch die besonders klaren topographischen Muster hervorgerufen werden, die sich den Sprechern im gebirgigen Gelände als Ankerpunkte anbieten. Andererseits zeichnet sich Südtirol gegenüber dem Oberrheingebiet durch eine außerordentlich hohe Sprachbewusstheit seiner deutschsprachigen Bevölkerung aus. Diese ist durch ihren Minderheitenstatus sowie eine diglossische

\footnotetext{
10 In sieben Fällen handelt es sich dabei um Komposita mit dem Determinatum „,-tal“ (Pustertal, Sarntal, Eisacktal, Ahrntal, Ultental, Etschtal, Wipptal), während die Gebiete Vinschgau, Unterland, Passeier und Grödner zwar nicht (mehrheitlich) als „Tal“ bezeichnet werden, es sich jedoch geographisch um solche handelt.
} 
Dialekt-Standardkonstellation geprägt, d. h. die Sprecher wechseln je nach kommunikativer Situation zwischen einem mehr oder weniger ausgeglichenen Südtiroler Grunddialekt und der deutschen Standardsprache. Im alltäglichen Umgang zwischen den deutschsprachigen Südtirolern ist dabei der Dialekt - ähnlich wie in der deutschsprachigen Schweiz - das alleinige Kommunikationsmittel, während der Standard lediglich in der formalen und schriftlichen Kommunikation (und auch hier nicht immer) Verwendung findet. Die hohe Sprachbewusstheit und Dialektkompetenz der Südtiroler könnte somit ebenfalls ein Grund dafür sein, weshalb die subjektive Verortung der Dialektgebiete in so konsistenter Weise geschieht. Gegen diese Argumentation spricht hingegen wieder die Tatsache, dass die Sprecher die Dialektgebiete aus Sicht der klassischen Dialektologie konsistent „falsch“ verorten, denn aus dem Tirolischen Sprachatlas ist eine nach Tälern ausgerichtete Binnengliederung der Südtiroler Dialekte nicht ersichtlich. Letztlich ist also die Topographie ausschlaggebend für die subjektive Binnengliederung durch die Südtiroler Sprecher und weniger eine an objektiv beobachtbaren sprachlichen Phänomenen ausgerichtete Verortung.

\section{$6 \quad$ Fazit und Ausblick}

Die eingangs formulierte Fragestellung nach einem Zusammenhang topographischer Strukturen und subjektiver Dialektverortung kann durch die vorgestellten Ergebnisse positiv beantwortet werden: Im kleinen Raum orientieren sich die Sprecher in erster Linie an naturräumlichen Objekten, und zwar umso mehr, je profilierter diese in der Landschaft sind. Dies geht aus dem Vergleich zwischen den beiden hier untersuchten, in topographischer Hinsicht sehr unterschiedlichen Gebieten deutlich hervor. Die einzige räumliche Kategorie, die alle anderen überragt, ist die nationale Grenze.

Das Ergebnis unserer Vergleichsstudie soll gleichzeitig einen Beitrag zur übergeordneten Frage leisten, nach welchen Prinzipien generell mentale Dialekträume konstruiert werden. Für eine allgemeine Typologie ist es an dieser Stelle sicherlich noch zu früh, da zu diesem Zweck eine größere Menge und vor allem Vielfalt an empirischen Daten notwendig wäre. Zur Beantwortung der Frage, ob topographische Bezüge tatsächlich nur auf der kleinräumigen Ebene bedeutsam sind, wäre es beispielsweise notwendig, Studien in größeren Untersuchungsgebieten (etwa auf das Gebiet eines Nationalstaates bezogen) mit ausgeprägter Topographie durchzuführen. Wie allerdings bisherige Forschungsergebnisse aus der deutschsprachigen Schweiz ${ }^{11}$ zeigen, stellt in diesem Gebiet die Kantonsstruktur (und somit eine politisch-administrative Kategorie) die alles überragende Ordnungsinstanz für die subjektive Gliederung der Dialektlandschaft dar, auch wenn mit Sicherheit davon ausgegangen werden kann, dass die Topographie eine alltagsweltliche Präsenz in den Köpfen der Sprecher besitzt und eine wichtige Grundlage für die geographische Einteilung der Schweiz darstellt.

Neben den geographischen und politischen Rahmenbedingungen spielt sicherlich noch ein weiterer Faktor bei der Dialektwahrnehmung eine Rolle, der im vorliegenden Beitrag weitgehend ausgeklammert wurde: die Dialekt/Standard-Konstellation und die damit verbundene Präsenz des Dialekts im Alltag der Sprecher. Während in beiden hier

\footnotetext{
11 Cf. dazu z. B. Christen $(1998,2010)$ sowie vorläufige Resultate einer jüngst von den Autoren dieses Artikels durchgeführten Studie zur subjektiven Dialektraumverortung in der Deutschschweiz.
} 
untersuchten Gebieten dialektnahe Sprachlagen sicherlich für einen beträchtlichen Teil der Sprecher von Bedeutung sind, wären für einen weiteren Vergleich subjektive Dialektdaten aus Gebieten interessant, in denen die Dialekte weitgehend abgebaut sind (z. B. in weiten Bereichen Norddeutschlands). Dieser Vergleich wird jedoch zukünftigen Forschungsvorhaben vorbehalten sein.

Geht man von der Annahme aus, dass ein Gebiet in irgendeiner Form geographisch verankert sein muss, um von den Sprechern als Dialektregion klassifiziert zu werden - und bisherige Studien belegen dies klar -, so deutet unsere Vergleichsstudie darauf hin, dass diese Klassifikationen mit umso größerer Übereinstimmung stattfinden, je prominenter die räumlich-strukturellen Charakteristika in Erscheinung treten.

\section{Literaturverzeichnis}

Anders, Christina Ada (2010): Wahrnehmungsdialektologie. Das Obersächsische im Alltagsverständnis von Laien. Berlin/New York: de Gruyter. (=Linguistik - Impulse \& Tendenzen 36).

Auer, Peter (2004): „Sprache, Grenze, Raum“. Zeitschrift für Sprachwissenschaft 23: 149179.

Auer, Peter et al. (2015): „Auswirkungen der Staatsgrenze auf die Sprachsituation im Oberrheingebiet (Frontière linguistique au Rhin Supérieur, FLARS)“. In: Kehrein, Roland/Lameli, Alfred/Rabanus, Stefan (eds.): Regionale Variation des Deutschen. Projekte und Perspektiven. Berlin/Boston, de Gruyter: 323-347.

Bach, Adolf (1934/1950): Deutsche Mundartforschung. Ihre Wege, Ergebnisse und Aufgaben. 2. Auflage. Heidelberg: Winter.

Bohnenberger, Karl (1928): „Über die Ostgrenze des Alemannischen“. Beiträge zur Geschichte der deutschen Sprache und Literatur 52: 217-291.

Christen, Helen (1998): Dialekt im Alltag. Eine empirische Untersuchung zur lokalen Komponente heutiger schweizerdeutscher Varietäten. Tübingen: Niemeyer. (= Germanistische Linguistik 201).

Christen, Helen (2010): „Was Dialektbezeichnungen und Dialektattribuierungen über alltagsweltliche Konzeptualisierungen sprachlicher Heterogenität verraten“. In: Anders, Christina Ada/Hundt, Markus/Lasch, Alexander (eds.): Perceptual Dialectology. Neue Wege der Dialektologie. Berlin/New York, de Gruyter: 269-290. (=Linguistik - Impulse \& Tendenzen 38).

Christen, Helen et al. (2015): „Ländere” : Die Urschweiz als Sprach(wissens)raum“. In: Kehrein, Roland/Lameli, Alfred/Rabanus, Stefan (eds.): Regionale Variation des Deutschen. Projekte und Perspektiven. Berlin/Boston, de Gruyter: 621-643.

Cukor-Avila, Patricia et al. (2012): “Texas - It's Like a Whole Nuther Country': Mapping Texans' Perceptions of Dialect Variation in the Lone Star State". Texas Linguistics Forum 55 (Proceedings of the Twentieth Annual Symposium about Language and Society - Austin, April 13-15, 2012): 10-19.

ESRI (s. n.) (2016): What is GIS? www.esri.com/what-is-gis. [01.05.2016].

Evans, Betsy E. (2011): “'Seattletonian' to 'Faux Hick': Perceptions of English in Washington State". American Speech 86/4: 383-413. 
Haag, Karl (1898): Die Mundarten des oberen Neckar- und Donaulandes (Schwäbischalemannisches Grenzgebiet: Baarmundarten). Reutlingen: Hutzler.

Hansen-Morath, Sandra/Stoeckle, Philipp (2014): „Regionaldialekte im alemannischen Dreiländereck - ,objektive“ und ,subjektive“ Perspektiven“. In: Bergmann, Pia et al. (eds.): Sprache im Gebrauch: räumlich, zeitlich, interaktional. Festschrift für Peter Auer. Heidelberg, Winter: 175-192. (= OraLingua 9).

Hundt, Markus/Palliwoda, Nicole/Schröder, Saskia (2015): „Wahrnehmungsdialektologie Der deutsche Sprachraum aus der Sicht linguistischer Laien. Exemplarische Ergebnisse des Kieler DFG-Projekts“'. In: Kehrein, Roland/Lameli, Alfred/Rabanus, Stefan (eds.): Regionale Variation des Deutschen. Projekte und Perspektiven. Berlin/Boston, de Gruyter: 585620.

Inoue, Fumino (1996): “Subjective Dialect Division in Great Britain”. American Speech 71/2: $142-161$.

Klausmann, Hubert (1990): „Staatsgrenze als Sprachgrenze? Zur Entstehung einer neuen Wort- und Sprachgebrauchsgrenze am Oberrhein“. Germanistische Linguistik 101-103: 193-215.

Kleene, Andrea (2015): „Mental Maps des Bairischen und seiner Grenzen“. In: Elmentaler, Michael/Hundt, Markus/Schmidt, Jürgen Erich (eds.): Deutsche Dialekte. Konzepte, Probleme, Handlungsfelder. Akten des 4. Kongresses der Internationalen Gesellschaft für Dialektologie des Deutschen (IGDD). Stuttgart, Steiner: 323-340. (=Zeitschrift für Dialektologie und Linguistik, Beihefte 158).

Klein, Karl Kurt/Schmitt, Ludwig Erich/Kühebacher, Egon (1965-1971): Tirolischer Sprachatlas. Marburg: Elwert. (= Deutscher Sprachatlas. Regionale Sprachatlanten 3).

König, Werner (2001): „Der nördliche Lech als Sprachgrenze“. In: Naturwissenschaftlicher Verein für Schwaben e. V. (ed.): Der nördliche Lech. Lebensraum zwischen Augsburg und Donau. 2. Auflage. Augsburg, Wißner: 45-54.

Kremer, Ludger (1984): „Die niederländisch-deutsche Staatsgrenze als subjektive Dialektgrenze“. Driemaandelijkse Bladen 36: 76-83.

Lameli, Alfred/Purschke, Christoph/Kehrein, Roland (2008): „Stimulus und Kognition. Zur Aktivierung mentaler Raumbilder". Linguistik online 35: 55-86.

Lynch, Kevin (1960): The image of the city. Cambridge: M.I.T. Press.

Maurer, Friedrich (1942): Oberrheiner, Schwaben, Südalemannen. Räume und Kräfte im geschichtlichen Aufbau des deutschen Südwestens. Strassburg: Hünenburg.

Montgomery, Chris (2006): Northern English Dialects: A Perceptual Approach. Unpublished $\mathrm{PhD}$ dissertation, University of Sheffield.

Montgomery, Chris/Stoeckle, Philipp (2013): “Geographic information systems and perceptual dialectology: a method for processing draw-a-map data”. Journal of Linguistic Geography 1: 52-85.

Paul, Hermann (1880/1975): Prinzipien der Sprachgeschichte. 9. Auflage. Tübingen: Niemeyer.

Pickl, Simon (2013): Probabilistische Geolinguistik. Geostatistische Analysen lexikalischer Variation in Bayerisch-Schwaben. Stuttgart: Steiner. (=Zeitschrift für Dialektologie und Linguistik, Beihefte 154).

Preston, Dennis R. (1982): "Perceptual dialectology. Mental maps of United States dialects from a Hawaiian perspective". Working Papers in Linguistics 14/2: 5-49. 
Preston, Dennis R. (1993): "Folk Dialect Maps". In: Glowka, Arthur Wayne/Lance, Donald M. (eds.): Language Variation in North American English. Research and Teaching. New York, Modern Language Association of America: 105-118.

Preston, Dennis R. (1999): “Introduction”. In: Preston, Dennis R. (ed.): Handbook of Perceptual Dialectology. Volume 1. Amsterdam/Philadelphia, Benjamins: xxiii-xl.

Preston, Dennis R. (2002): "Language with an Attitude". In: Chambers, Jack K./Trudgill, Peter/Schilling-Estes, Natalie (eds.): The Handbook of Language Variation and Change. Oxford, Blackwell: 40-66.

Purschke, Christoph (2011): Regionalsprache und Hörerurteil. Grundzüge einer perzeptiven Variationslinguistik. Stuttgart: Steiner. (= Zeitschrift für Dialektologie und Linguistik, Beihefte 149).

Schifferle, Hans-Peter (1990): „Badisches und schweizerisches Alemannisch am Hochrhein“. Germanistische Linguistik 101-103: 315-340.

Seidelmann, Erich (1989): „Der Hochrhein als Sprachgrenze“. In: Putschke, Wolfgang/Veith, Werner/Wiesinger, Peter (eds.): Dialektgeographie und Dialektologie. Günter Bellmann zum 60. Geburtstag von seinen Schülern und Freunden. Marburg, Elwert: 57-88.

Sibata, Takesi (1959/1999): “Consciousness of Dialect Boundaries”. In: Preston, Dennis R. (ed.): Handbook of Perceptual Dialectology. Volume 1. (Original 1959 erschienen als "Hôgen kyôkai no ishiki". Gengo Kenkyû 36, 1-30). Amsterdam/Philadelphia, Benjamins: $39-62$.

Spiekermann, Helmut/Hohenstein, Line-Marie (2016): „Mehrsprachigkeit an der Grenze. Sprachwissen und -wahrnehmung im Emsland und in der Grafschaft Bentheim“. Niederdeutsches Jahrbuch 138: 99-118.

Stoeckle, Philipp (2014): Subjektive Dialekträume im alemannischen Dreiländereck. Hildesheim: Olms. (= Deutsche Dialektgeographie 112).

Streck, Tobias (2012): „Dialektareale in Baden-Württemberg. Dialektometrische Analysen spontansprachlicher Daten aus Südwestdeutschland und Überlegungen zum Zusammenhang von objektiven Dialektgrenzen und mentalen Raumkonzepten“. In: Hansen, Sandra et al. (eds.): Dialectological and Folk Dialectological Concepts of Space. Current Methods and Perspectives in Sociolinguistic Research on Dialect Change. Berlin/Boston, de Gruyter: 232-249. (= linguae et litterae 17).

Weichhart, Peter (2008): Entwicklungslinien der Sozialgeographie. Stuttgart: Steiner. (= Sozialgeographie kompakt 1).

Weijnen, Antonius A. (1946): „De grenzen tussen de Oost-Noordbrabantse dialecten onderling“. In: Weijnen, Antonius Angelus/Renders, J. M./van Ginneken, Jacobus Johannes Antonius (eds.): Oost-Noordbrabantse dialectproblemen. Amsterdam, Noord-Hollandsche uitgeversmij: $1-15$. 